\title{
Association between sustainable food choice motives during purchasing and dietary patterns in French adults
}

\author{
B. Allès ${ }^{1}$, S. Péneau ${ }^{1}$, E. Kesse-Guyot ${ }^{1}$, J. Baudry ${ }^{1}$, S. Hercberg ${ }^{1,2}$ and C. Méjean ${ }^{1}$ \\ ${ }^{1}$ Université Paris 13, COMUE Sorbonne-Paris-Cité, Equipe de Recherche en Epidémiologie Nutritionnelle (EREN), \\ Centre d'Epidémiologie et Biostatistiques Paris Nord, Inserm (U1153), Inra (U1125) and Département de Santé \\ Publique, Hôpital Avicenne, Bobigny, France
}

During the last decade, there was an increasing concern about sustainability issues in consumer food choice motives ${ }^{(1)}$. However, the association between these motives and dietary patterns has not been studied. This cross-sectional study aimed to investigate the relationships between sustainable food choice motives during purchasing and dietary pattern in a large sample of French adults.

Food choice motives were collected in 31,846 adults participating at the Nutrinet-Santé study, using a validated 63 items questionnaire ${ }^{(2)}$ gathered into 9 dimension scores: ethics and environment, traditional and local production, taste, price, environmental limitations, health, convenience, innovation and absence of contaminants. Dietary intakes were assessed using at least three 24-h food records and classified into food groups according to the French dietary guidelines. Three dietary patterns were derived through factoranalysis using principal component analysis. Multiple linear regression models were performed to assess the associations between dietary patterns and dimension scores.

Taste, health, ethics and environment dimensions had the highest mean scores. The three dietary patterns reflected a "healthy diet", "traditional diet" and "western diet".

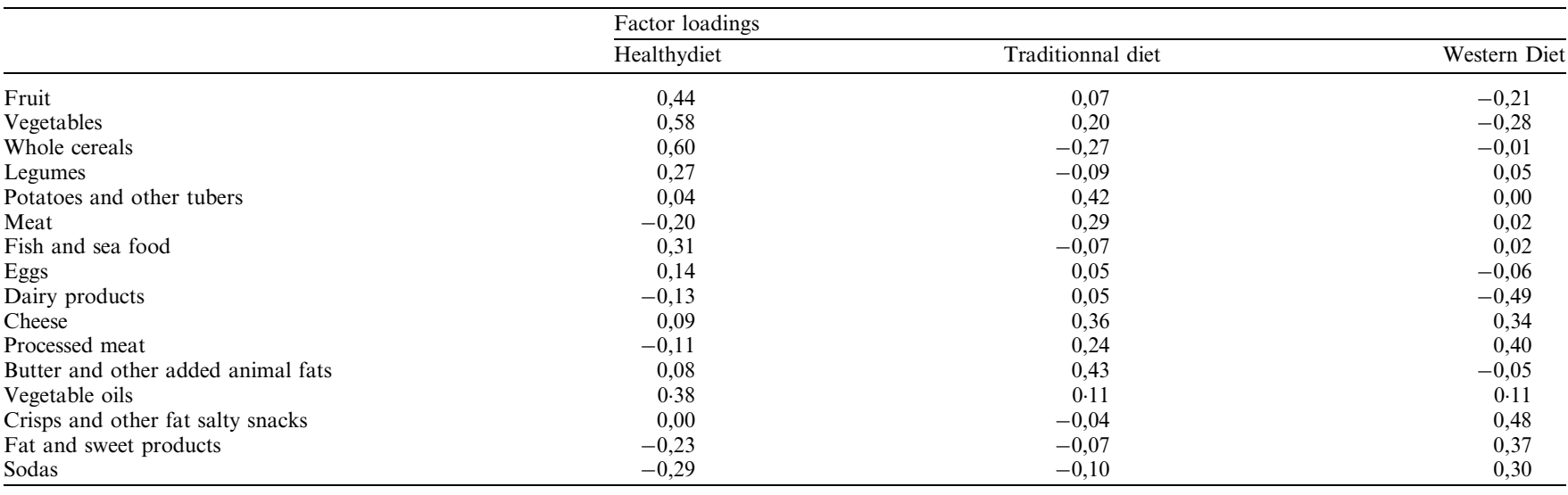

9 food groups included in the factor analysis are not shown: alcoholic beverages, non alcoholic and non sweet beverages, starchy refined foods, sauces, puddings, margarine, sweets, sweet and fat breakfast cereals and low fat and sugar breakfast cereal

The Healthy pattern (table) had higher factor loadings for food groups composed by plant-based products (except fish and seafood and eggs) whereas the two other dietary patterns had higher factor loadings for food groups composed by animal-based products.

Individuals more concerned by environmental limitations $\left(\beta_{\text {women }}=0 \cdot 18,95 \% \mathrm{CI}=0 \cdot 15-0 \cdot 20, \beta_{\mathrm{men}}=0 \cdot 20,95 \% \mathrm{CI}=0 \cdot 15-0 \cdot 25\right)$, ethics and environment (women only, $\beta=0.05,95 \% \mathrm{CI}=0.02-0.08)$, absence of contaminants (women only, $\beta=0.04,95 \% \mathrm{CI}=$ 0.01-0.07), local production (women only, $\beta=0.08,95 \% \mathrm{CI}=0.05-0.11$ ) but also health (women only) and innovation (men only), and those less interested in price, were more likely to have a "healthy diet". The traditional and western diets were less likely to be associated with sustainable food choice motives dimension scores.

This study highlighted that sustainable motives in food purchases were associated with a healthier diet characterized by lower consumptions of animal products.

1. Verain MCD, Dagevos H \& Antonides G (2015) Appetite In press.

2. Sautron V, Péneau S, Camilleri GM et al. (2015) Appetite 87, 90-7. 